\title{
Comparison of acute kidney injury with radial vs. femoral access for patients undergoing coronary catheterization: An updated meta-analysis of 46,816 patients
}

\author{
CHANG WANG, WEIWEI CHEN, MING YU and PING YANG
}

\begin{abstract}
Department of Cardiology, China-Japan Union Hospital of Jilin University, Jilin Provincial Precision Medicine Key Laboratory for Cardiovascular Genetic Diagnosis, Jilin Provincial Engineering Laboratory for Endothelial Function and Genetic Diagnosis of Cardiovascular Disease, Jilin Provincial Molecular Biology Research Center for Precision Medicine of Major Cardiovascular Disease, Jilin Provincial Cardiovascular Research Institute, Changchun, Jilin 130028, P.R. China
\end{abstract}

Received March 3, 2020; Accepted July 17, 2020

DOI: $10.3892 /$ etm.2020.9170

\begin{abstract}
Trans-radial access for percutaneous coronary intervention or angiography has gained popularity amongst interventional cardiologists. Radial access is also thought to reduce the incidence of acute kidney injury (AKI) in the immediate post-operative period. The purpose of the present study was to perform a comprehensive updated systematic review and meta-analysis comparing the incidence of AKI following the radial vs. femoral route of coronary catheterization. An electronic literature search of the PubMed, BioMed Central, Scopus, Cochrane Central Register of Controlled Trials and Google Scholar databases up to 1st January 2020 was performed. A total of 14 studies were included, 2 of which were randomized controlled trials (RCTs), and 6 studies utilized propensity score matching. Comparison of the data of 21,479 patients in the radial group and 25,337 patients in the femoral group indicated a reduced incidence of AKI with the radial route [odds ratio (OR):0.66,95\% CI: 0.54-0.81, $\mathrm{P}<0.0001$, $\left.\mathrm{I}^{2}=74 \%\right]$. Similar results were obtained with sub-group analyses for RCTs (OR: 0.87, 95\% CI: 0.77-0.98, $\mathrm{P}=0.02, \mathrm{I}^{2}=0 \%$ ), retrospective studies (OR: 0.57, 95\% CI: 0.36-0.90, $\mathrm{P}=0.02$, $\mathrm{I}^{2}=86 \%$ ) and propensity score-matched studies (OR: $0.63,95 \%$ CI: $0.48-0.83, \mathrm{P}=0.0009, \mathrm{I}^{2}=45 \%$ ). Multivariable-adjusted ORs of AKI for the radial vs. femoral route were extracted
\end{abstract}

Correspondence to: Dr Ping Yang, Department of Cardiology, China-Japan Union Hospital of Jilin University, Jilin Provincial Precision Medicine Key Laboratory for Cardiovascular Genetic Diagnosis, Jilin Provincial Engineering Laboratory for Endothelial Function and Genetic Diagnosis of Cardiovascular Disease, Jilin Provincial Molecular Biology Research Center for Precision Medicine of Major Cardiovascular Disease, Jilin Provincial Cardiovascular Research Institute, 126 Xiantai Street, Changchun, Jilin 130028, P.R. China

E-mail: pyang122@163.com

Key words: percutaneous coronary intervention, coronary angiography, contrast-induced nephropathy, vascular access from non-RCTs and pooled for a meta-analysis, which also demonstrated similar results (OR: $0.70,95 \%$ CI: $0.57-0.88$, $\mathrm{P}=0.002, \mathrm{I}^{2}=70 \%$ ). Within the limitations of the study, the present results indicate that, as compared to femoral access, the use of trans-radial access for coronary catheterization is associated with a significantly reduced incidence of AKI. A reduction of AKI by $\sim 34 \%$ may be expected with the use of radial access.

\section{Introduction}

Acute kidney injury (AKI) is a potential complication that may increase morbidity and mortality after any percutaneous coronary intervention (PCI) or angiography procedure (1). Several risk factors for AKI following coronary catheterization have been recognized and they may be broadly divided into patient-associated or procedure-related factors. Patient characteristics, including old age, hypotension, diabetes mellitus, pre-existing renal impairment, history of renal transplantation, anemia and use of nephrotoxic drugs are known to significantly increase the risk of post-procedural AKI. Similarly, procedural factors, including contrast media properties, contrast media volume and number of procedures within $72 \mathrm{~h}$ also influence the probability of AKI $(2,3)$.

The incidence of AKI following the use of contrast media has been reported to be $0-24 \%$ (2). Although in most cases, AKI is transient, irreversible renal injury may occur in a proportion of patients, leading to progressive kidney damage, which may require prolonged hospitalization and result in long-term adverse effects (4). Of the several strategies to reduce contrast-induced $\mathrm{AKI}$, volume expansion has been most commonly used. However, the benefits of prophylactic fluid replacement have been questioned by recent reports, which suggest a limited benefit in high-risk patients (5). Drugs including $\mathrm{N}$-acetylcysteine and bicarbonate have also been used, but with limited evidence of a benefit (6).

To reduce the incidence of AKI following coronary catheterization, the association of vascular access and the incidence of AKI has been explored in the past decade (7). Trans-radial access for PCI or angiography, which has gained 
popularity amongst interventional cardiologists, is thought to reduce the incidence of AKI in the immediate post-operative period. To date, several studies have compared AKI following radial vs. femoral access in patients undergoing coronary catheterization but with variable results (8-10). Certain meta-analyses have been published comparing AKI with the two vascular access sites $(1,7)$. In the most recent metaanalysis, Andò et al (7) pooled results from 10 studies and explored the association between radial access and AKI. The authors, however, only compiled data on the absolute number of AKI events from the included studies. Despite several retrospective studies utilizing multivariate regression analysis to adjust for baseline characteristics for reporting outcomes, no attempt has been made to date to collate such data and analyze the overall evidence on the incidence of AKI with radial vs. femoral access, to the best of our knowledge. In addition, with further studies published $(8,11,12)$, there is a requirement for an updated review and meta-analysis on this important topic. Therefore, the purpose of the present study was to perform a comprehensive updated review and meta-analysis comparing AKI following the radial vs. femoral route of coronary catheterization.

\section{Materials and methods}

Inclusion criteria. The Population, Intervention, Comparison, Outcome and Study design model was used to select studies for this systematic review (13). Retrospective, as well as prospective studies, were included in the analysis. To be included in the review, studies were required to have been conducted on adult patients (Population) requiring coronary catheterization for any reason (PCI or coronary angiography). Studies were required to compare the radial route (Intervention) with the femoral route (Comparison) for catheterization. The Outcome of the study was required to be the incidence of AKI. The definition of AKI was as per the included study. Studies not reporting relevant data, studies reporting duplicate data, single-arm studies, case series, case reports, non-English language studies and review articles were excluded. The Preferred Reporting Items for Systematic Reviews and Meta-analyses guidelines were followed during the conduct of this systematic review (13).

Search strategy. A computerized literature search of the PubMed, BioMed Central, Scopus, Cochrane Central Register of Controlled Trials and Google scholar databases was performed. The search was conducted by two reviewers independently (CW and WC). Databases were screened from inception up to 1st January 2020. MeSH terms, as well as free-text keywords, were used in the literature search. The entire search protocol, as well as the number of results obtained from the PubMed database, is presented in Table SI. The entries obtained were screened by their titles and abstracts for each database. Potentially relevant articles were then extracted and subsequently screened by their full text. Both reviewers assessed individual studies based on the inclusion criteria and resolved any disagreement by discussion. After screening, the bibliography of included studies, as well as review articles on the subject, were hand-searched for any additional references.
Data extraction and risk of bias assessment. Using a pre-formatted abstraction form, the reviewers extracted data from the included studies. Details including the first author name, publication year, sample size, demographic details, baseline characteristics of the study sample, definition of AKI, contrast volume and incidence of AKI were extracted.

The risk of bias of randomized controlled trials (RCTs) was assessed using the Cochrane Collaboration risk assessment tool for RCTs (14). Every study was assessed regarding the following domains: Random sequence generation, allocation concealment, blinding of participants and personnel, blinding of outcome assessment, incomplete outcome data, selective reporting and other biases. The risk of a bias assessment tool for non-randomized studies was used to assess non-RCTs (15). Studies were rated as having low risk, high risk, or unclear risk of bias for the following: Selection of participants, confounding variables, intervention measurements, blinding of outcome assessment, incomplete outcome data and selective outcome reporting.

Statistical analysis. Considering the methodological heterogeneity of the included studies, a random-effects model was used to calculate the pooled effect size for all analyses. The incidence of AKI between the radial and femoral routes was compared using the generic inverse-variance method of DerSimonian and Laird. Odds ratios (ORs) with 95\% CI were calculated. Retrospective studies, case-control studies using propensity score matching and RCTs were grouped separately for calculating effect sizes (primary meta-analysis). A sub-group analysis was also conducted for non-RCTs based on the definition of AKI. Where available, multivariable-adjusted ORs of AKI for radial vs. femoral route were also obtained from non-RCTs. All study-specific ORs were then combined using inverse variance-weighted averages of logarithmic ORs (secondary meta-analysis). A sensitivity analysis was performed to assess the influence of each study on the pooled effect size for both the primary and secondary meta-analysis. Heterogeneity was assessed using the $\mathrm{I}^{2}$ statistic. $\mathrm{I}^{2}$ values of $25-50 \%$ represented low, values of $50-75 \%$ medium and $>75 \%$ represented substantial heterogeneity. Visual inspection of funnel plots was performed to explore possible small-study and publication biases. The software 'Review Manager' [version 5.3; Nordic Cochrane Centre (Cochrane Collaboration)] was used for the meta-analysis.

\section{Results}

Study selection and characteristics. After a comprehensive literature search, 19 studies were found eligible for full-text analysis (Fig. 1). A total of 5 studies were then excluded, as 2 reported data $(16,17)$ from an already included RCT and the other 3 studies (18-20) did not report on the incidence of AKI. A total of 14 studies were included in this systematic review and meta-analysis (8-12,21-29).

The characteristics of the included studies are presented in Table I. Two studies $(8,10)$ were RCTs, while the remaining studies were retrospective in nature. Furthermore, six retrospective studies utilized propensity score matching to harmonize the baseline characteristics of the study participants $(9,12,22,25,27,29)$. In the included studies, participants 


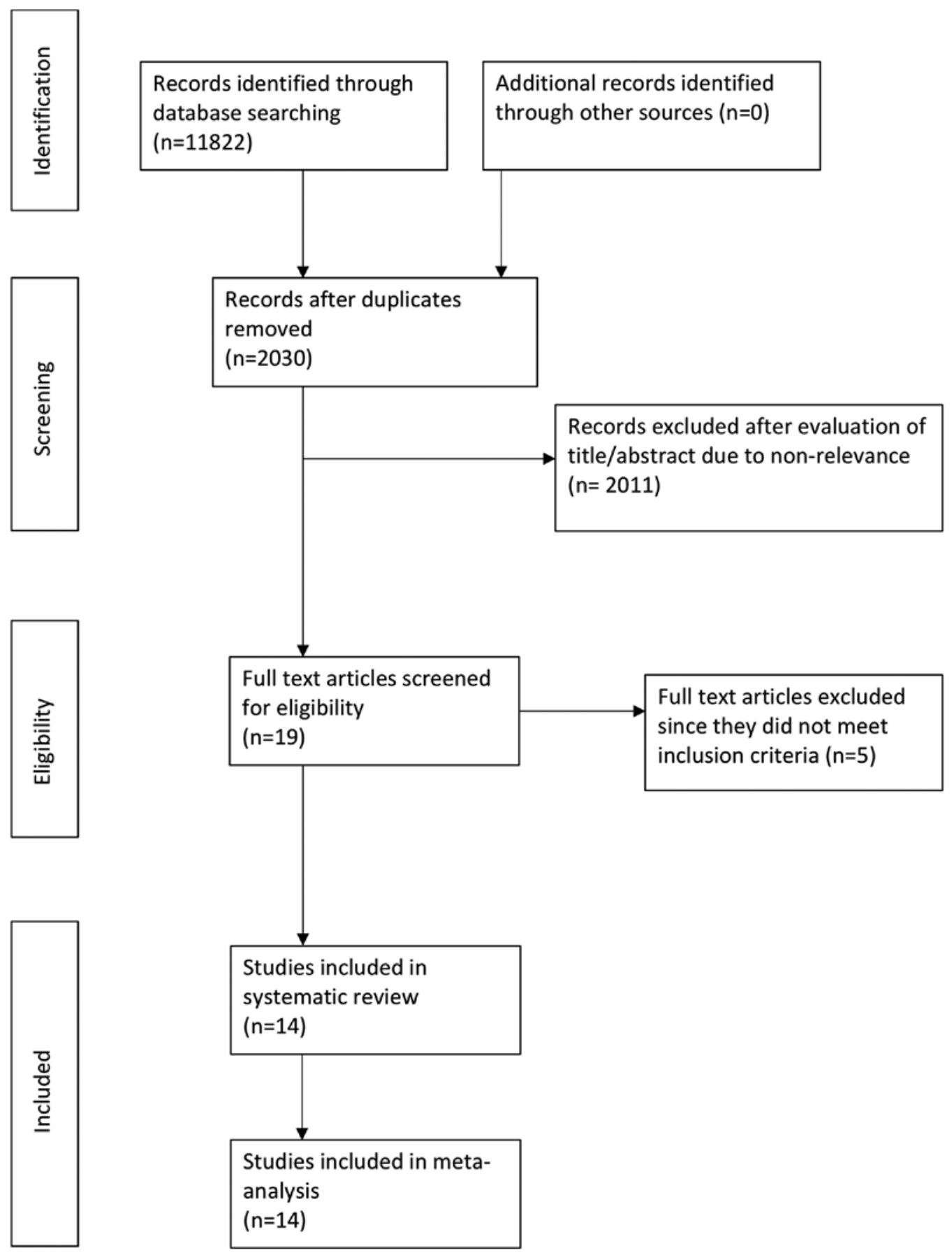

Figure 1. Flow chart for the search and inclusion of studies in the present meta-analysis.

underwent coronary catheterization for a variety of reasons. There was a wide variation in the sample size of included studies ranging from 30 participants per group to a maximum of 8,857 patients per group. The mean age of the study population was $>60$ years in all included studies. AKI was either defined as creatinine levels of $\geq 0.3$ or $\geq 0.5 \mathrm{mg} / \mathrm{dl}$ postprocedure. However, the time-lapse of AKI was not coherent amongst the included studies. A total of five studies measured creatinine levels within $48 \mathrm{~h}$ of the procedure, while 4 studies defined AKI as an increase in creatinine levels within $72 \mathrm{~h}$ of catheterization. The mean contrast volume was not reported in 4 studies $(8,9,12,25)$. Baseline patient details of included studies are presented in Table II.
Primary meta-analysis. The absolute incidence of AKI was extracted from individual studies and pooled for the present meta-analysis. The pooled effect size of the two RCTs included in this review indicated that the radial route of coronary catheterization significantly reduced the incidence of AKI as compared to the femoral route (OR: $0.87,95 \%$ CI: 0.77-0.98, $\mathrm{P}=0.02, \mathrm{I}^{2}=0 \%$; Fig. 2). Data of a total of 22,196 patients in 6 studies were compared utilizing propensity score matching. Sub-group analysis of these studies also revealed significantly reduced AKI with the radial route of catheterization (OR: 0.63, 95\% CI: 0.48-0.83, $\mathrm{P}=0.0009, \mathrm{I}^{2}=45 \%$; Fig. 2). Finally, analysis of 6 retrospective studies wherein the two groups were not matched for baseline characteristics also demonstrated a 


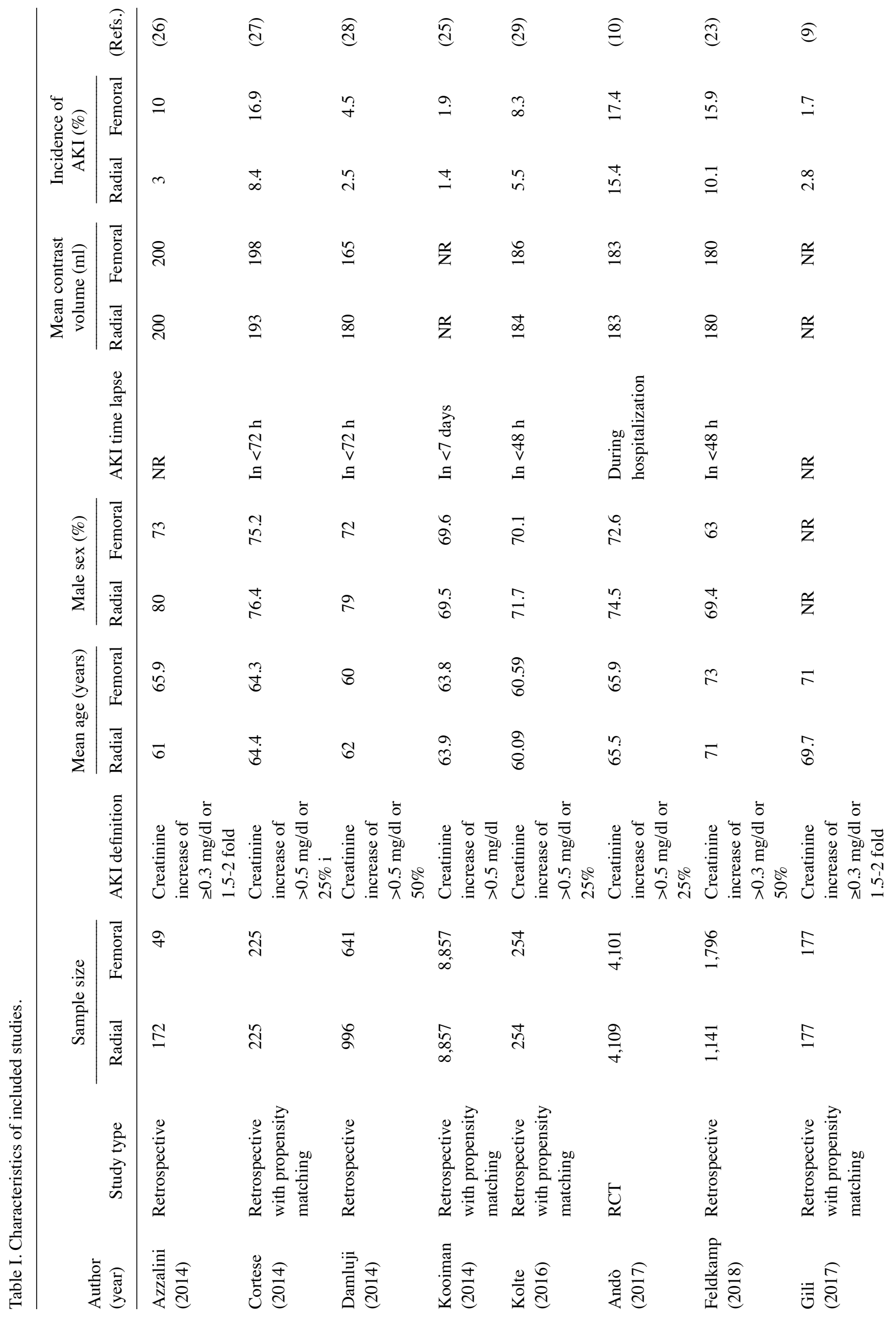




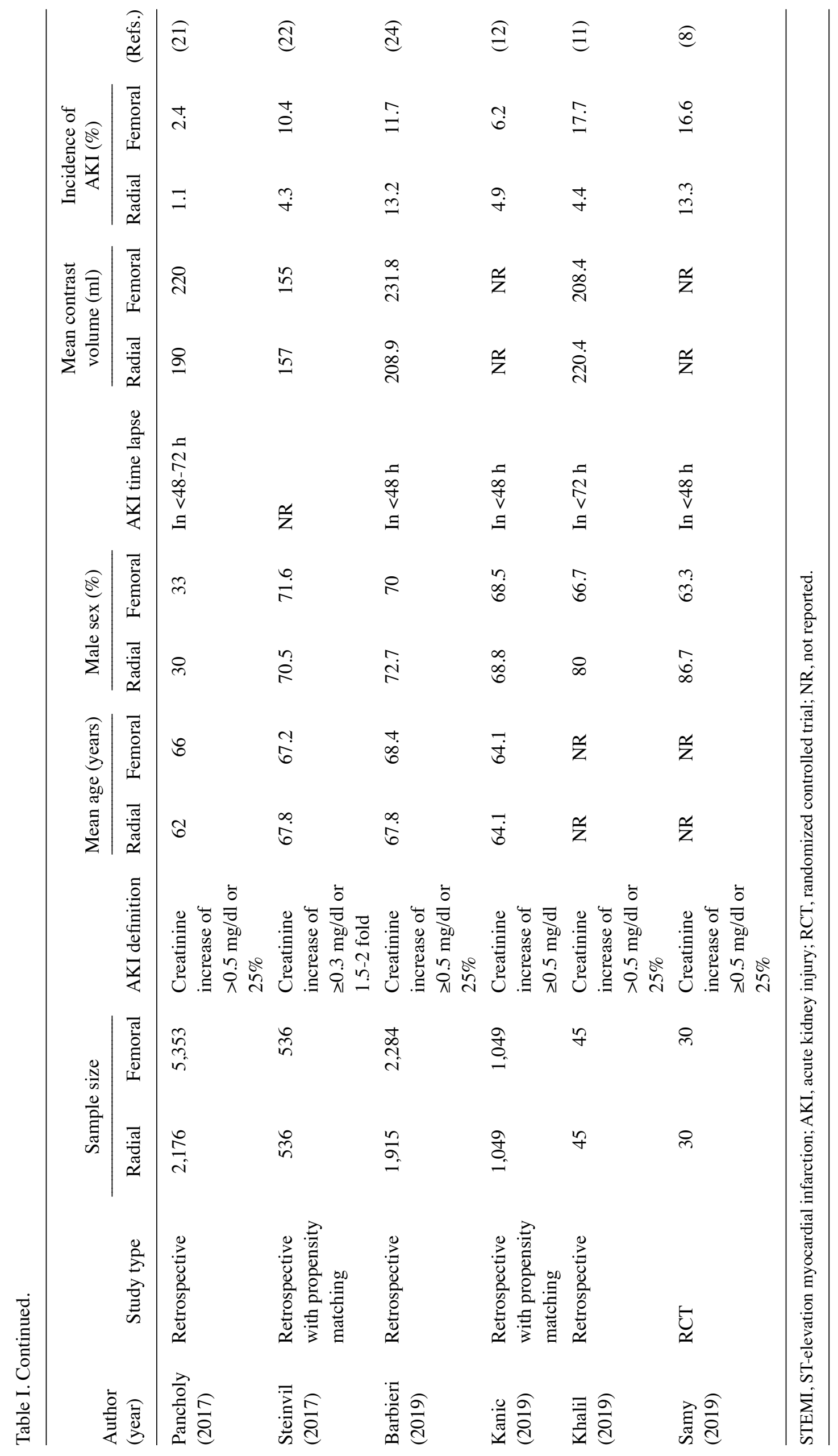




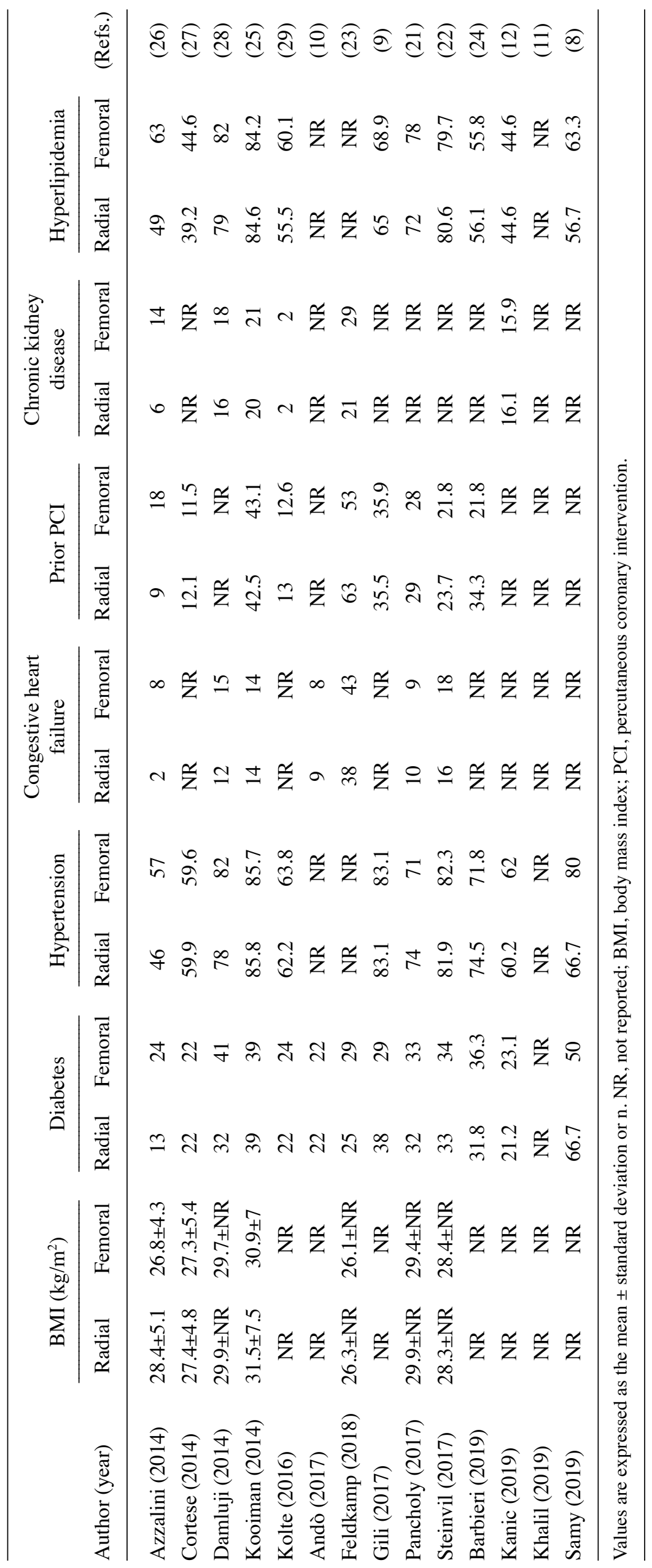




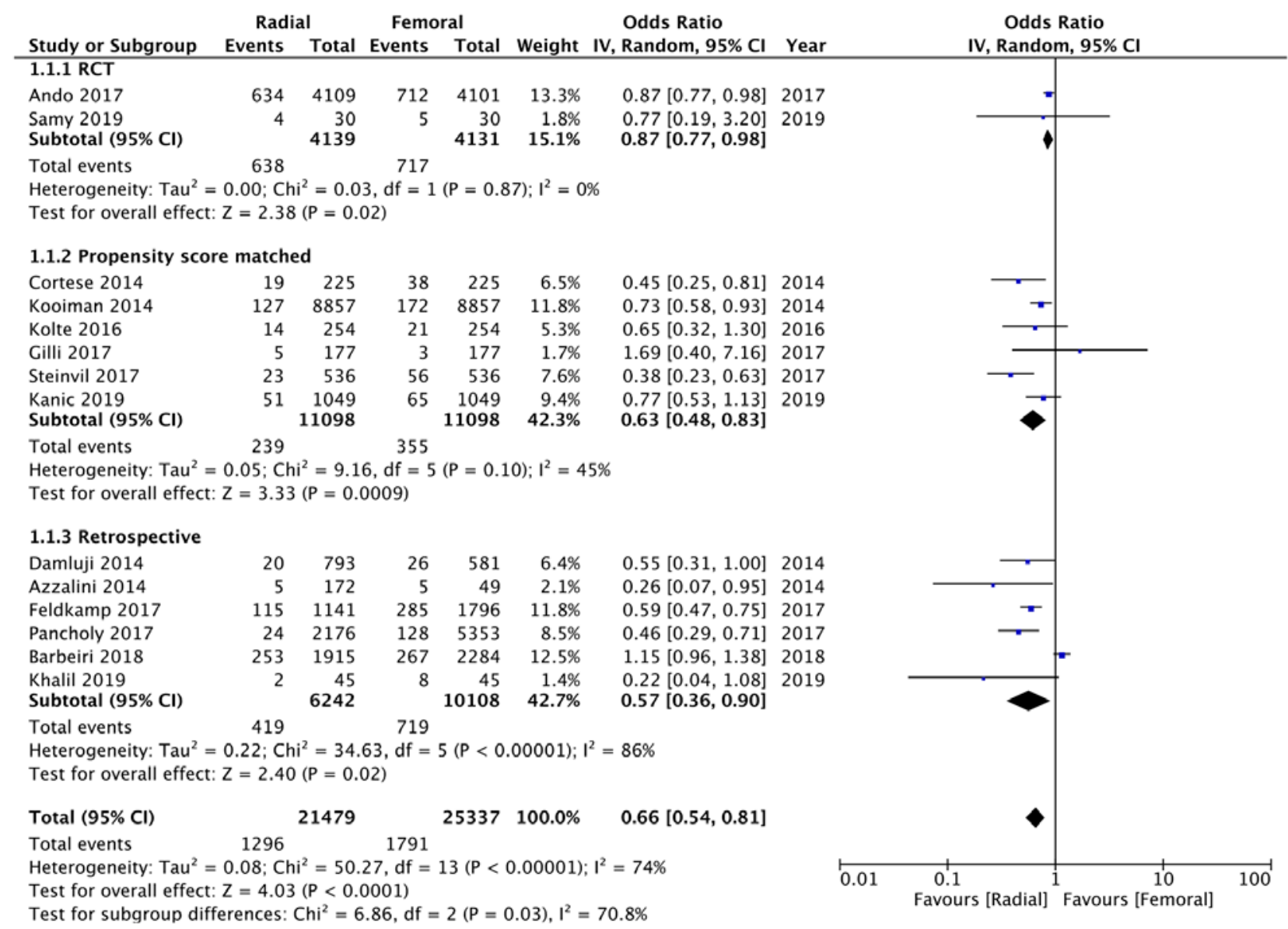

Figure 2. Forest plot for the incidence of acute kidney injury for the radial vs. femoral route with sub-group analysis based on study type. IV, inverse variance; df, degrees of freedom; RCT, randomized controlled trial.

significant benefit of radial access in reducing AKI (OR: 0.57, 95\% CI: 0.36-0.90, $\mathrm{P}=0.02, \mathrm{I}^{2}=86 \%$; Fig. 2). Overall, when the data of the 21,479 patients in the radial group and 25,337 patients in the femoral group were compared, the analysis indicated a $35 \%$ reduced incidence of AKI with the radial route (OR: $0.66,95 \% \mathrm{CI}$ : $0.54-0.81, \mathrm{P}<0.0001, \mathrm{I}^{2}=74 \%$; Fig. 2). A certain amount of asymmetry was identified on visual inspection of the funnel plot (Fig. 3).

A subgroup analysis was performed for retrospective studies based on the diagnostic criteria for AKI. Two retrospective studies defined AKI as an increase of $\geq 0.3 \mathrm{mg} / \mathrm{dl}$ of creatinine, while the remaining four considered $>0.5 \mathrm{mg} / \mathrm{dl}$ of creatinine as AKI. On subgroup analysis, there was a significant advantage of the radial route when AKI was defined as $\geq 0.3 \mathrm{mg} / \mathrm{dl}$ of creatinine (OR: $0.51,95 \% \mathrm{CI}: 0.27-0.95, \mathrm{P}=0.03$, $\mathrm{I}^{2}=33 \%$ ), but not when the criterion was $\geq 0.5 \mathrm{mg} / \mathrm{dl}$ (OR: 0.60 , 95\% CI: $0.31-1.16, \mathrm{P}=0.13, \mathrm{I}^{2}=86$; Fig. 4). On the contrary, in studies utilizing propensity-scored matched groups, the present analysis indicated no difference in AKI between the two groups when AKI was defined as $\geq 0.3 \mathrm{mg} / \mathrm{dl}$ of creatinine (OR: 0.68 , 95\% CI: $0.17-2.81, \mathrm{P}=0.06, \mathrm{I}^{2}=72 \%$ ), but a significantly reduced odds of AKI when it was defined as $>0.5 \mathrm{mg} / \mathrm{dl}$ of creatinine (OR: 0.70, 95\% CI: 0.59-0.84, $\mathrm{P}=0.0001, \mathrm{I}^{2}=0 \%$; Fig. 5).

Secondary analysis. Multivariable regression analysis was performed by 9 studies included in the review (12,21-25,27-29).

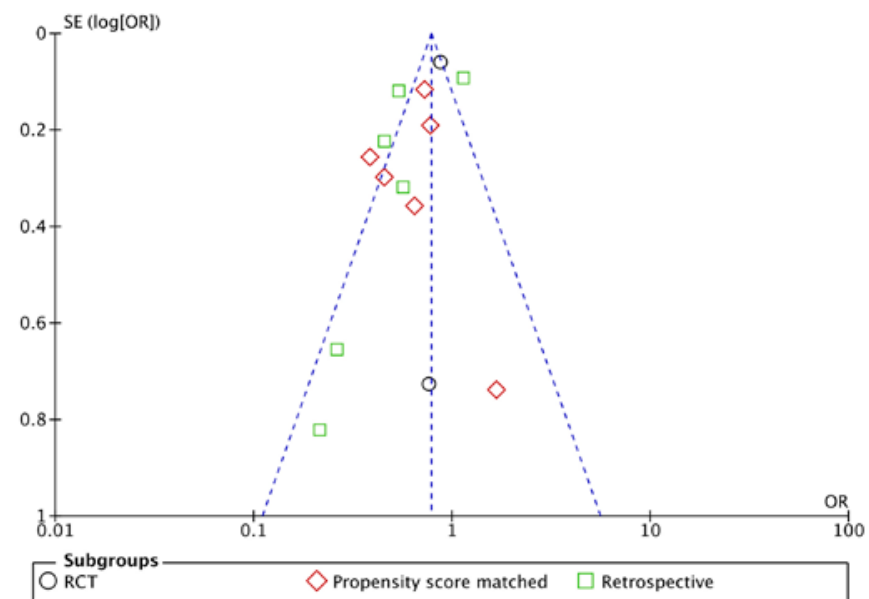

Figure 3. Funnel plot for the incidence of acute kidney injury for the radial vs. femoral route based on study type. SE, standard error; OR, odds ratio; RCT, randomized controlled trial.

Adjusted ORs were extracted from these studies and pooled for a meta-analysis. The results from the secondary meta-analysis indicated a $30 \%$ reduced odds of AKI with the radial route of catheterization as compared to the femoral route (OR: $0.70,95 \% \mathrm{CI}: 0.57-0.88, \mathrm{P}=0.002, \mathrm{I}^{2}=70 \%$; Fig. 6). The funnel plot of the analysis demonstrated minimal 


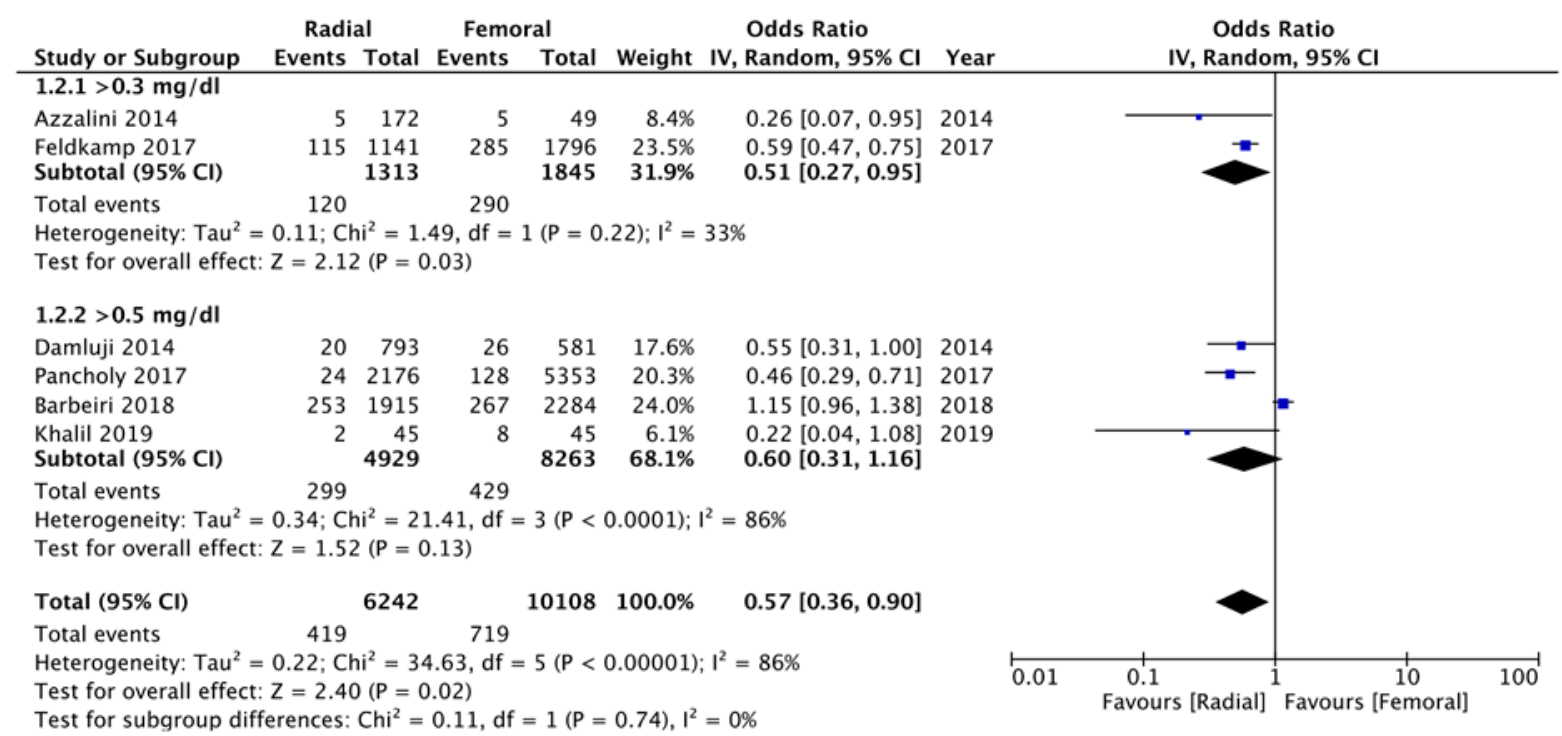

Figure 4. Forest plot of retrospective studies for the incidence of AKI with sub-group analysis based on the definition of AKI. AKI, acute kidney injury; IV, inverse variance; df, degrees of freedom.

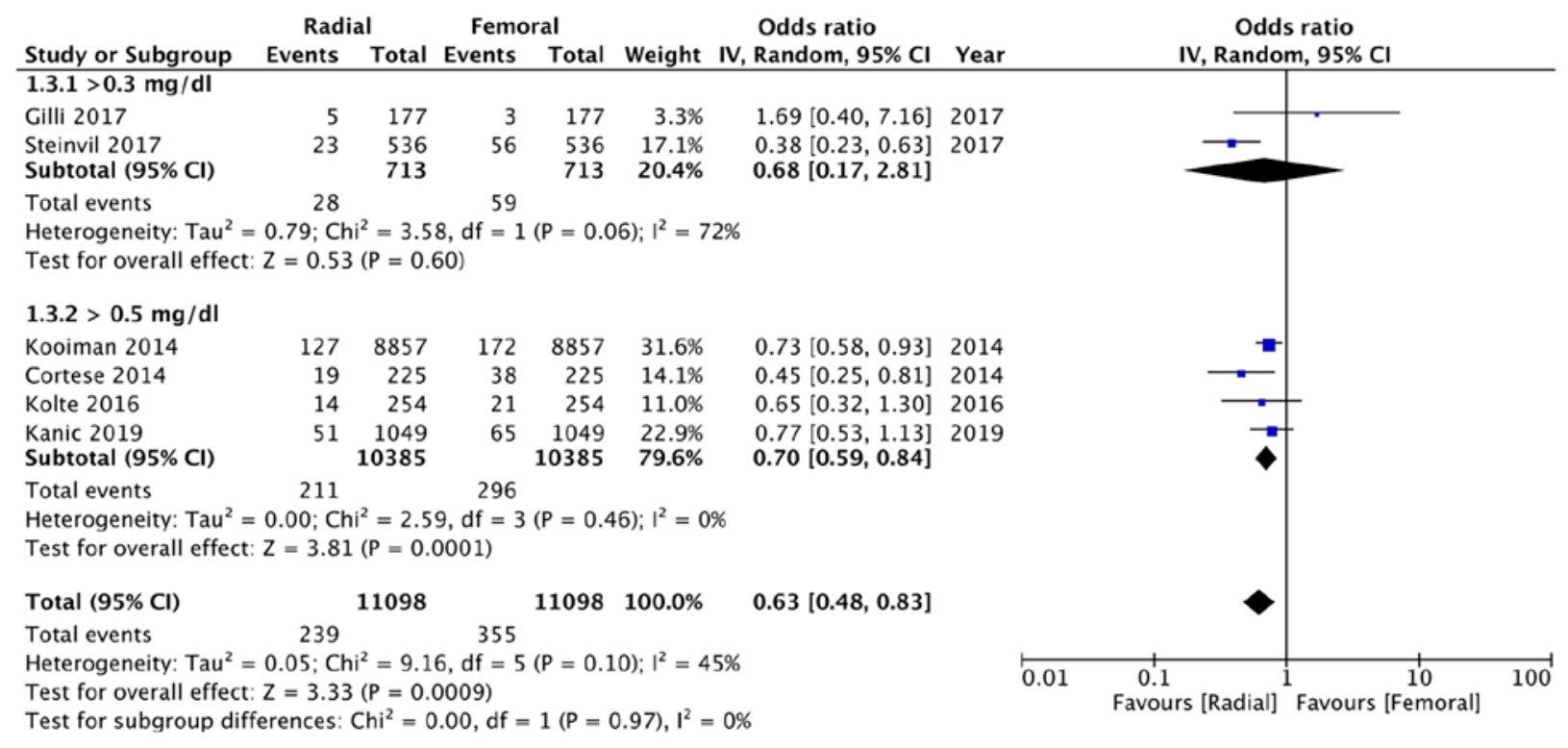

Figure 5. Forest plot of propensity score-matched studies for the incidence of AKI with sub-group analysis based on the definition of AKI. AKI, acute kidney injury; IV, inverse variance; df, degrees of freedom.

asymmetry, with only two of the nine studies outside the funnel plot (on either side) (Fig. 7).

Sensitivity analysis and risk of bias assessment. Studies were sequentially excluded from both the primary and secondary meta-analysis to assess the effect of individual studies on the pooled effect size. Detailed results of the sensitivity analysis are presented in Table III. For the primary meta-analysis, there was no change in the significance of results with the exclusion of any study in the propensity score-matched group. However, for retrospective studies, when the studies of Feldkamp et al (23) and Pancholy et al (21) were excluded, there was no significant difference in the incidence of AKI between the two groups. For the secondary meta-analysis, there was no change in the significance of results after the exclusion of any study. The authors' judgement of risk of bias of included studies is presented in Table SII. For RCTs, sufficient information on allocation concealment and blinding of personnel and participants was not available. For retrospective studies, confounding variables were not taken into account in all studies and none reported blinding of outcomes. Overall, the risk of bias in the included studies was moderate.

\section{Discussion}

Coronary angiography and PCI are important diagnostic and management tools for treating coronary artery disease (CAD). With the increased incidence of CAD and improvements in 
Odds ratio

Study or Subgroup log[Odds Ratio] SE Weight IV, Random, 95\% CI Year

Cortese 2014

Damluji 2014

Kooiman 2014

Kolte 2016

Feldkamp 2017

Pancholy 2017

Steinvil 2017

Barbeiri 2018

Kanic 2019

Total $(95 \% \mathrm{Cl})$

Heterogeneity: $\mathrm{Tau}^{2}=0.07 ; \mathrm{Chi}^{2}=26.92, \mathrm{df}=8(\mathrm{P}=0.0007) ; \mathrm{I}^{2}=70 \%$
Test for overall effect: $\mathrm{Z}=3.14(\mathrm{P}=0.002)$

$\begin{array}{llllll}-0.5108 & 0.2133 & 10.9 \% & 0.60[0.40,0.91] & 2014\end{array}$

$\begin{array}{lllll}-0.4005 & 0.377 & 5.9 \% & 0.67[0.32,1.40] & 2014\end{array}$

$\begin{array}{lllll}-0.2744 & 0.1039 & 15.6 \% & 0.76[0.62,0.93] & 2014\end{array}$

$\begin{array}{lllll}-0.1744 & 0.3299 & 7.0 \% & 0.84[0.44,1.60] & 2016\end{array}$

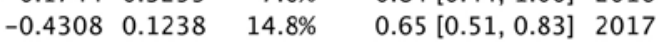

$\begin{array}{llllll}-0.5621 & 0.2488 & 9.5 \% & 0.57[0.35,0.93] & 2017\end{array}$

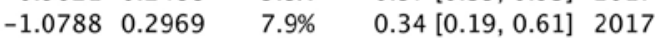

$\begin{array}{llllll}0.174 & 0.1149 & 15.2 \% & 1.19[0.95,1.49] & 2018\end{array}$

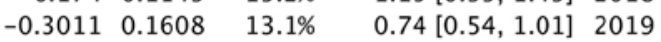

$100.0 \% \quad 0.70[0.57,0.88]$

Odds ratio

IV, Random, $95 \% \mathrm{CI}$

Figure 6. Forest plot of multivariate-adjusted odds ratios for the incidence of acute kidney injury with radial vs. femoral route. IV, inverse variance; df, degrees of freedom.

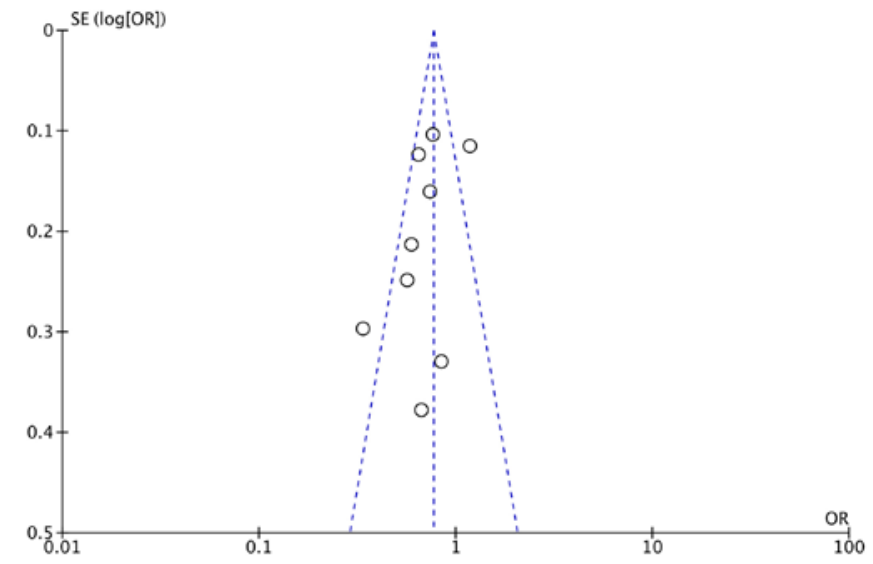

Figure 7. Funnel plot of multivariate-adjusted odds ratios for the incidence of acute kidney injury with the radial vs. femoral route. SE, standard error; OR, odds ratio.

stent technology, a significantly larger number of patients are undergoing coronary catheterization (30). Concurrently, there is a growing need to limit post-procedural complications and enhance clinical outcomes. The development of AKI after coronary catheterization is multifactorial, including both patient and procedural factors (3). The purpose of the present study was to gather evidence on whether the vascular access site influences the incidence of AKI in such patients. Analysis of 14 studies including a total of 46,816 patients revealed that compared with femoral access, radial access for PCI or coronary angiography may reduce the incidence of AKI by an estimated 34\% (range: $19-46 \%)$.

It is important to note that the current meta-analysis pooled a heterogeneous group of studies involving 2 RCTs, 6 retrospective studies without matching the baseline study population and 6 studies utilizing propensity-scored matched data. Individually, 7 studies $(8,9,11,12,24,28,29)$ did not report any association between the vascular access site and AKI, while the remaining studies demonstrated a significantly reduced incidence of AKI with the radial route (10,21-23,25-27). Among the studies not reporting any difference between the radial and the femoral route were 1 RCT, 3 retrospective studies and 3 propensity-scored matched studies.
Several factors, including age, hydration status, preexisting diseases, medications and procedural characteristics, may influence AKI in a patient undergoing coronary catheterization. It is therefore essential that baseline patient and procedural factors are matched when assessing the difference in AKI between the radial and the femoral route. RCTs are designed to provide unbiased results, as they reduce the influence of confounding factors and have lower chances of systematic errors (31). In the case of deficiency of RCTs, the evidence is frequently generated from retrospective data using either propensity score matching or multivariate logistic regression adjustment (32). Propensity score matching is a quasi-empirical correction strategy that attempts to decrease bias of treatment estimates in non-randomized studies by forming matched sets of study and control groups $(32,33)$. On the other hand, regression analysis is a commonly used statistical tool to assess the impact of an independent variable on a dichotomous dependent variable (33). Both techniques have been widely utilized in observation studies and have their own set of advantages and drawbacks. The superiority of one technique over the other is difficult to judge, as neither of them take into account unknown confounding factors that may affect the outcome (32). Therefore, to provide a broad perspective on the results of different study types and different matching techniques, different sets of analyses were performed in the present study. On subgroup analysis based on study type, the pooled analysis of the two RCTs indicated a 23\% reduced incidence of AKI with the radial route of access. However, as the recent RCT by Samy et al (8) included just 30 patients in each group, the results were particularly influenced by the larger AKI-MATRIX trial (10). Similar results were obtained on the pooling of data from retrospective non-adjusted studies, albeit with a wider $95 \%$ CI. The pooled analysis indicated a $43 \%$ reduced odds of AKI varying from 10 to $64 \%$. The wide range and superior results were probably due to the influence of non-adjusted confounding factors in these studies. Analysis of propensity score-matched studies revealed a 37\% reduced incidence of AKI (range, $17-52 \%$ ) with the use of the radial access site. These figures closely concurred with the pooled multivariate-adjusted ORs of 9 studies demonstrating a 30\% (range, 12-43\%) reduced chance of AKI with radial as compared to the femoral route for coronary catheterization. Near identical results obtained 
Table III. Results of the sensitivity analysis.

A, Propensity score-matched studies

\begin{tabular}{|c|c|c|c|c|}
\hline Study excluded & OR $(95 \% \mathrm{CI})$ & P-value & $\mathrm{I}^{2}(\%)$ & (Refs.) \\
\hline Cortese et al & $0.66(0.50-0.89)$ & 0.006 & 46 & (27) \\
\hline Kooiman et al & $0.59(0.41-0.86)$ & 0.005 & 48 & $(25)$ \\
\hline Kolte et al & $0.62(0.45-0.86)$ & 0.004 & 56 & $(29)$ \\
\hline Gili et al & $0.61(0.47-0.80)$ & 0.003 & 47 & (9) \\
\hline Steinvil et al & $0.71(0.60-0.85)$ & 0.002 & 0 & $(22)$ \\
\hline Kanic et al & $0.59(0.41-0.84)$ & 0.004 & 52 & (12) \\
\hline
\end{tabular}

B, Retrospective studies

\begin{tabular}{|c|c|c|c|c|}
\hline Study excluded & OR $(95 \% \mathrm{CI})$ & P-value & $\mathrm{I}^{2}(\%)$ & (Refs.) \\
\hline Damluji et al & $0.57(0.34-0.96)$ & 0.04 & 88 & (28) \\
\hline Azzalini et al & $0.62(0.38-0.99)$ & 0.05 & 87 & (26) \\
\hline Feldkamp et al & $0.53(0.28-1.01)^{\mathrm{a}}$ & 0.06 & 84 & (23) \\
\hline Pancholy et al & $0.61(0.36-1.01)^{\mathrm{a}}$ & $0.05 \# 6$ & 86 & (21) \\
\hline Barbieri et al & $0.54(0.45-0.66)$ & $<0.00001$ & 0 & (24) \\
\hline Khalil et al & $0.61(0.38-0.97)$ & 0.04 & 88 & (11) \\
\hline
\end{tabular}

C, Studies reporting multivariate-adjusted OR

\begin{tabular}{|c|c|c|c|c|}
\hline Study excluded & OR $(95 \% \mathrm{CI})$ & P-value & $\mathrm{I}^{2}(\%)$ & (Refs.) \\
\hline Cortese et al & $0.72(0.57-0.91)$ & 0.006 & 73 & (27) \\
\hline Damluji et al & $0.71(0.56-0.89)$ & 0.003 & 74 & (28) \\
\hline Kooiman et al & $0.69(0.52-0.90)$ & 0.007 & 74 & $(25)$ \\
\hline Kolte et al & $0.69(0.55-0.88)$ & 0.002 & 74 & (29) \\
\hline Feldkamp et al & $0.71(0.55-0.91)$ & 0.008 & 72 & (23) \\
\hline Pancholy et al & $0.72(0.57-0.91)$ & 0.006 & 72 & (21) \\
\hline Steinvil et al & $0.76(0.62-0.92)$ & 0.006 & 63 & (22) \\
\hline Barbieri et al & $0.67(0.58-0.77)$ & $<0.00001$ & 15 & (24) \\
\hline Kanic et al & $0.69(0.54-0.89)$ & 0.005 & 74 & (12) \\
\hline
\end{tabular}

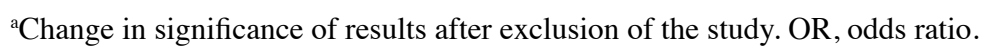

with both propensity score and multivariate regression data in the present analysis provided strong evidence that the use of the radial access site significantly reduces the incidence of post-procedural AKI.

Another important difference between the included studies was the definition of AKI. Contrast-induced AKI as defined by the Kidney Disease: Improving Global Outcomes workgroup in 2012 is an absolute increase in serum creatinine of $\geq 0.3 \mathrm{mg} / \mathrm{dl}$ within $48 \mathrm{~h}$ or an increase of $\geq 25 \%$ from baseline (34). Alternatively, the Contrast Media Safety Committee of the European Society of Urogenital Radiology defines AKI as $\geq 0.5 \mathrm{mg} / \mathrm{dl}$ or $\geq 25 \%$ of the baseline increase in serum creatinine within $72 \mathrm{~h}$ of contrast exposure (35). To evaluate the influence of the definition of AKI on study outcomes, studies defining AKI as a creatinine increase of $\geq 0.3$ or $\geq 0.5 \mathrm{mg} / \mathrm{dl}$ were grouped. In the propensity score-matched studies, there was no difference between the radial and femoral access site when AKI was defined as $\geq 0.3 \mathrm{mg} / \mathrm{dl}$, but a significant advantage of radial access was observed when AKI was defined as $\geq 0.5 \mathrm{mg} / \mathrm{dl}$ of creatinine. This may indicate that there may be no difference in the milder form of AKI with the use of either access site but a higher grade of AKI may be reduced with the radial route. However, due to limited studies in the analysis and contrasting results of the subgroup analysis of retrospective studies, no strong conclusions can be drawn from this difference. 
Two important reasons are thought to reduce the incidence of AKI with trans-radial access. Bleeding events and access site complications are known risk factors for the development of AKI following coronary catheterization. As bleeding events are significantly reduced with the use of radial access, it is likely that the radial route provides a reno-protective effect by limiting renal hypoperfusion (24). Furthermore, it is also postulated that the incidence of athero-embolization is higher with femoral as compared to radial vascular access. Since the catheter passed via the femoral route comes in contact with thoracic and abdominal aortic atheroma, the catheter may scrape of plaque material, resulting in embolization to the renal vasculature (36). Since the radial route completely avoids these arteries, the risk of renal embolization is therefore significantly reduced.

The results of the present study should be interpreted considering the following limitations. First, only 2 RCTs were included in the analysis and majority studies were retrospective in nature. The lack of RCTs reduced the level of evidence and increased bias of the results. Despite using propensity score matching and multivariate regression adjustment, the influence of all confounding factors on the study outcomes is not completely negated. Furthermore, there was methodological heterogeneity between the included studies concerning factors such as the study population, baseline patient characteristics, and definition and time-lapse of AKI. Of note, the exact definition of AKI varied among the included studies. This may have skewed the results of the present analysis. Finally, data about reno-protective measures, including the use of hydration, was not available from the included studies. To what extent other AKI preventive measures were utilized in the radial and femoral groups is also not known.

As compared to the previous meta-analysis (7), the present study was able to incorporate 4 further studies $(8,11,12,24)$; it included the data of a total of 46,816 patients and is therefore a significant update. In addition to the subgroup analysis based on study type, the influence of the definition of AKI on the pooled effect size was also analyzed. Adjusted ORs from retrospective studies were also subjected to a meta-analysis to provide comprehensive evidence on the subject. Except for the retrospective studies, the results of the present analysis were robust in terms of effect size and direction after the sensitivity analysis.

Within the limitations of the present study, the results indicated that, as compared to femoral access, the use of trans-radial access for coronary catheterization significantly reduced the incidence of AKI. A reduction of AKI by $~ 34 \%$ may be expected with the use of radial access. In addition, there may be no difference in the milder form of AKI with the use of either access site and only a higher grade of AKI may be reduced with the radial route. Further large-scale RCTs are required to strengthen the current evidence.

\section{Acknowledgements}

Not applicable.

\section{Funding}

No funding was received.

\section{Availability of data and materials}

The datasets used and/or analyzed during the present study are available from the corresponding author on reasonable request.

\section{Authors' contributions}

CW conceived and designed the study. CW and WC collected the data and performed the literature search. MY and PY performed the meta-analysis. CW was involved in the writing of the manuscript. All authors have read and approved the final manuscript.

\section{Ethics approval and consent to participate}

Not applicable.

\section{Patient consent for publication}

Not applicable.

\section{Competing interests}

The authors declare that they have no competing interests.

\section{References}

1. Andò G, Costa F, Boretti I, Trio O and Valgimigli M: Benefit of radial approach in reducing the incidence of acute kidney injury after percutaneous coronary intervention: A meta-analysis of 22,108 patien. Int J Cardiol 179: 309-311, 2015.

2. Hossain MA, Costanzo E, Cosentino J, Patel C, Qaisar H, Singh V, Khan T, Cheng JS, Asif A and Vachharajani TJ: Contrast-induced nephropathy: Pathophysiology, risk factors, and prevention. Saudi J Kidney Dis Transpl 29: 1-9, 2018.

3. Mehran R, Aymong ED, Nikolsky E, Lasic Z, Iakovou I, Fahy M, Mintz GS, Lansky AJ, Moses JW, Stone GW, et al: A simple risk score for prediction of contrast-induced nephropathy after percutaneous coronary intervention: Development and initial validation. J Am Coll Cardiol 44: 1393-1399, 2004.

4. Rihal CS, Textor SC, Grill DE, Berger PB, Ting HH, Best PJ, Singh M, Bell MR, Barsness GW, Mathew V, et al: Incidence and prognostic importance of acute renal failure after percutaneous coronary intervention. Circulation 105: 2259-2264, 2002.

5. Nijssen EC, Rennenberg RJ, Nelemans PJ, Essers BA, Janssen MM, Vermeeren MA, Ommen VV and Wildberger JE: Prophylactic hydration to protect renal function from intravascular iodinated contrast material in patients at high risk of contrast-induced nephropathy (AMACING): A prospective, randomised, phase 3 , controlled, open-label, non-inferiority trial. Lancet 389: 1312-1322, 2017.

6. Feldkamp T and Kribben A: Contrast media induced nephropathy: Definition, incidence, outcome, pathophysiology, risk factors and prevention. Minerva Med 99: 177-196, 2008.

7. Andò $G$, Gragnano F, Calabrò $P$ and Valgimigli M: Radial vs femoral access for the prevention of acute kidney injury (AKI) after coronary angiography or intervention: A systematic review and meta-analysis. Catheter Cardiovasc Interv 92: E518-E526, 2018.

8. Samy NI, Walaafareed, Ahmed AAS and Osama M: Contrast Induced Nephropathy after radial or femoral access for invasive management of acute coronary syndrome. World J Cardiovasc Dis 9: $572-583,2019$.

9. Gili S, D'Ascenzo F, Di Summa R, Conrotto F, Cerrato E, Chieffo A, Boccuzzi G, Montefusco A, Ugo F, Omedé P, et al: Radial versus femoral access for the treatment of left main lesion in the era of second-generation drug-eluting stents. Am J Cardiol 120: 33-39, 2017.

10. Andò G, Cortese B, Russo F, Rothenbühler M, Frigoli E, Gargiulo G, Briguori C, Vranckx P, Leonardi S, Guiducci V, et al: Acute kidney injury after radial or femoral access for invasive acute coronary syndrome management: AKI-MATRIX. J Am Coll Cardiol, May 11, 2017 (Online ahead of print). 
11. Khalil WA, Abdelhameed MG, Elmenshawy MD and Elhelaly A Serum cystatin $\mathrm{c}$ and renal dysfunction in transradial versus transfemoral approaches in primary percutaneous coronary intervention. J Indian Coll Cardiol 9: 88-94, 2019.

12. Kanic V, Kompara G, Suran D, Tapajner A, Naji FH and Sinkovic A: Acute kidney injury in patients with myocardial infarction undergoing percutaneous coronary intervention using radial versus femoral access. BMC Nephrol 20: 28, 2019.

13. Moher D, Liberati A, Tetzlaff J and Altman DG; PRISMA Group: Preferred reporting items for systematic reviews and meta-analyses: The PRISMA statement. PLoS Med 6: e1000097, 2009.

14. Higgins JPT, Altman DG and Sterne JAC (eds): Cochrane statistical methods group and the cochrane bias methods group. Chapter 8: Assessing risk of bias in included studies. In: Cochrane Handbook for Systemic Reviews of Interventions, Version 5. The Cochrane Collaboration, 2011.

15. Kim SY, Park JE, Lee YJ, Seo HJ, Sheen SS, Hahn S, Jang BH and Son HJ: Testing a tool for assessing the risk of bias for nonrandomized studies showed moderate reliability and promising validity. J Clin Epidemiol 66: 408-414, 2013

16. Valgimigli M, Gagnor A, Calabró P, Frigoli E, Leonardi S, Zaro T, Rubartelli P, Briguori C, Andò G, Repetto A, et al: Radial versus femoral access in patients with acute coronary syndromes undergoing invasive management: A randomised multicentre trial. Lancet 385: 2465-2476, 2015.

17. Rothenbühler M, Valgimigli M, Odutayo A, Frigoli E, Leonardi S, Vranckx P, Turturo M, Moretti L, Amico F, Uguccioni L, et al: Association of acute kidney injury and bleeding events with mortality after radial or femoral access in patients with acute coronary syndrome undergoing invasive management: Secondary analysis of a randomized clinical trial. Eur Heart J 40: 1226-1232, 2019.

18. Alonzo A, Rigattieri S, Giovannelli F, Di Russo C, Sciahbasi A, Berni A and Volpe M: Transfemoral approach with systematic use of FemoSeal ${ }^{\mathrm{TM}}$ closure device compared to transradial approach in primary angioplasty. Catheter Cardiovasc Interv 87: 849-854, 2016.

19. Ratib K, Mamas MA, Anderson SG, Bhatia G, Routledge $H$ De Belder M, Ludman PF, Fraser D and Nolan J; British Cardiovascular Intervention Society and the National Institute for Cardiovascular Outcomes Research: Access site practice and procedural outcomes in relation to clinical presentation in 439,947 patients undergoing percutaneous coronary intervention in the United kingdom. JACC Cardiovasc Interv 8: 20-29, 2015.

20. Mamas MA, Ratib K, Routledge H, Neyses L, Fraser DG, de Belder M, Ludman PF and Nolan J; British Cardiovascular Intervention Society and the National Institute for Cardiovascular Outcomes Research: Influence of arterial access site selection on outcomes in primary percutaneous coronary intervention: Are the results of randomized trials achievable in clinical practice? JACC Cardiovasc Interv 6: 698-706, 2013.

21. Pancholy MS, Skelding K, Scott T, Blankenship J and Pancholy SB Effect of access site choice on acute kidney injury after percutaneous coronary intervention. Am J Cardiol 120: 2141-2145, 2017.

22. Steinvil A, Garcia-Garcia HM, Rogers T, Koifman E, Buchanan K, Alraies MC, Torguson R, Pichard AD, Satler LF, Ben-Dor I and Waksman R: Comparison of propensity score-matched analysis of acute kidney injury after percutaneous coronary intervention with transradial versus transfemoral approaches. Am J Cardiol 119: 1507-1511, 2017

23. Feldkamp T, Luedemann M, Spehlmann ME, Freitag-Wolf S, Gaensbacher J, Schulte K, Bajrovic A, Hinzmann D, Hippe HJ, Kunzendorf U, et al: Radial access protects from contrast media induced nephropathy after cardiac catheterization procedures. Clin Res Cardiol 107: 148-157, 2018.
24. Barbieri L, Verdoia M, Suryapranata H and De Luca G; Novara Atherosclerosis Study Group (NAS): Impact of vascular access on the development of contrast induced nephropathy in patients undergoing coronary angiography and/or percutaneous coronary intervention. Int J Cardiol 275: 48-52, 2019.

25. Kooiman J, Seth M, Dixon S, Wohns D, LaLonde T, Rao SV and Gurm HS: Risk of acute kidney injury after percutaneous coronary interventions using radial versus femoral vascular access: Insights from the blue cross blue shield of michigan cardiovascular consortium. Circ Cardiovasc Interv 7: 190-198, 2014.

26. Azzalini L, Khan R, Al-Hawwas M, Hatem R, Fortier A, L'Allier PL and Ly HQ: Effect of radial-to-femoral access crossover on adverse outcomes in primary percutaneous coronary intervention. Am J Cardiol 114: 1165-1173, 2014

27. Cortese B, Sciahbasi A, Sebik R, Rigattieri S, Alonzo A, Silva-Orrego P, Belloni F, Seregni RG, Giovannelli F, Tespili M, et al: Comparison of risk of acute kidney injury after primary percutaneous coronary interventions with the transradial approach versus the transfemoral approach (from the PRIPITENA urban registry). Am J Cardiol 114: 820-825, 2014.

28. Damluji A, Cohen MG, Smairat R, Steckbeck R, Moscucci M and Gilchrist IC: The incidence of acute kidney injury after cardiac catheterization or PCI: A comparison of radial vs. femoral approach. Int J Cardiol 173: 595-597, 2014

29. Kolte D, Spence N, Puthawala M, Hyder O, Tuohy CP Davidson CB, Sheldon MW, Laskey WK and Abbott JD: Association of radial versus femoral access with contrast-induced acute kidney injury in patients undergoing primary percutaneous coronary intervention for ST-elevation myocardial infarction. Cardiovasc Revasc Med 17: 546-551, 2016.

30. Likosky DS, Van Parys J, Zhou W, Borden WB, Weinstein MC and Skinner JS: Association between medicare expenditure growth and mortality rates in patients with acute myocardial infarction: A comparison from 1999 through 2014. JAMA Cardiol 3: 114-122, 2018.

31. Burns PB, Rohrich RJ and Chung KC: The levels of evidence and their role in evidence-based medicine. Plast Reconstr Surg 128: 305-310, 2011.

32. Biondi-Zoccai G, Romagnoli E, Agostoni P, Capodanno D, Castagno D, D'Ascenzo F, Sangiorgi G and Modena MG: Are propensity scores really superior to standard multivariable analysis? Contemp Clin Trials 32: 731-740, 2011.

33. Baser O: Choosing propensity score matching over regression adjustment for causal inference: When, why and how it makes sense. J Med Econ 10: 379-391, 2007.

34. Kellum JA and Lameire N; KDIGO AKI Guideline Work Group: Diagnosis, evaluation, and management of acute kidney injury: A KDIGO summary (Part 1). Crit Care 17: 204, 2013.

35. Morcos SK, Thomsen HS and Webb JA: Contrast-media-induced nephrotoxicity: A consensus report. Contrast media safety committee, european society of urogenital radiology (ESUR). Eur Radiol 9: 1602-1013, 1999.

36. Scolari F, Ravani P, Gaggi R, Santostefano M, Rollino C, Stabellini N, Colla L, Viola BF, Maiorca P, Venturelli C, et al: The challenge of diagnosing atheroembolic renal disease: Clinical features and prognostic factors. Circulation 116: 298-304, 2007.

This work is licensed under a Creative Common Attribution-NonCommercial-NoDerivatives 4.0 International (CC BY-NC-ND 4.0) License. 